\section{Ausgezeichnet Preis für Dirk Einecke}

— Dr. med. Dirk Einecke, langjähriger Chefredakteur der MMW-Fortschritte der Medizin und Herausgeber von Springer Medizin, erhält in diesem Jahr den Walter-Trummert-Preis. Seit 1974 wird dieser Preis jährlich vom Vorstand der Vereinigung der Deutschen Medizinischen Fach- und Standespresse e.V. (VMWJ) an verdiente Medizinjournalisten verliehen. Mit dem im Andenken an den früh gestorbenen Medizinjournalisten Walter Trummert gestifteten Preis werden die besonderen Verdienste von Dr. Einecke um die medizinische Publizistik gewürdigt.

Prof. Hermann S. Füess/

\section{Umfrage zu Spiritual Care Teilnahme erbeten}

- Die European Association for Palliative Care (EAPC) ruft zur Teilnahme an ihrer Befragung zu Forschungsprioritäten im Bereich Spiritual Care auf. Im April 2011 hatte sie eine Taskforce zu Spiritual Care in der Palliativmedizin gegründet, die helfen soll, spirituelle Bedürfnisse von Patienten, ihren Familien und Begleitern zu identifizieren. Mit Ihrer Teilnahme an der Online-Befragung (http://www.eapcnet.eu), die nur einige Minuten dauert, helfen Sie bisher unbeachtete Aspekte der spirituellen Begleitung in den Fokus zu rücken und schon benannte Forschungsansätze zu priorisieren. Kim Jené

\section{Apothekenbetriebsordnung Neuordnung positiv}

\begin{abstract}
- Ambulante Palliativpatienten profitieren von der Novelle der Apothekenbetriebsordnung (ApBetrO), die mit den letzten Bundesratsänderungen im Juni in Kraft treten soll. Dem Bundesministerium für Gesundheit (BMG) zufolge helfen die von den Ländern eingebrachten Regelungen zur BetäubungsmittelVorratshaltung, die ambulante Palliativversorgung zu verbessern. Zudem hingen sie eng mit einer im Betäubungmittelrecht geplanten Fortentwicklung zusammen: Ärzte sollen in Ausnahmefällen ambulanten Palliativpatienten zur Überbrückung Betäubungsmittel überlassen dürfen.

Kim Jené
\end{abstract}

\section{Betreuung Sterbender \\ Ärzte fordern nationale Strategie}

\footnotetext{
- Die Charta zur Betreuung schwerstkranker und sterbender Patienten wurde im September 2010 beschlossen. Nun soll sie praktische Wirkung zeitigen. Dieses Ziel haben sich die drei Trägerorganisationen Bundesärztekammer, Deutsche Gesellschaft für Palliativmedizin und Deutscher Hospiz- und Palliativverband gesetzt.

Unter Beteiligung von 50 Verbänden, Ministerien und den beiden großen Kirchen wurde die Charta erarbeitet und im September 2010 beschlossen. In ihr plädieren die Beteiligten für bessere, vernetzte Versorgungsstrukturen und einen Ausbau der Aus-, Weiter- und Fortbildung. Bis Ende 2013 läuft die „Charta II“ genannte Projektphase, in der nun die Umsetzung der Ziele ansteht. Unter anderem soll eine nationale Strategie zur Betreuung schwerstkranker und sterbender Menschen in Deutschland angestoßen werden. Dazu wollen die Initiatoren 5.000 Unterstützer finden. Finanziell getragen wird das Projekt von der Robert Bosch-Stiftung, dem Bundesfamilienministerium und den drei Trägerorganisationen. Florian Staeck
}

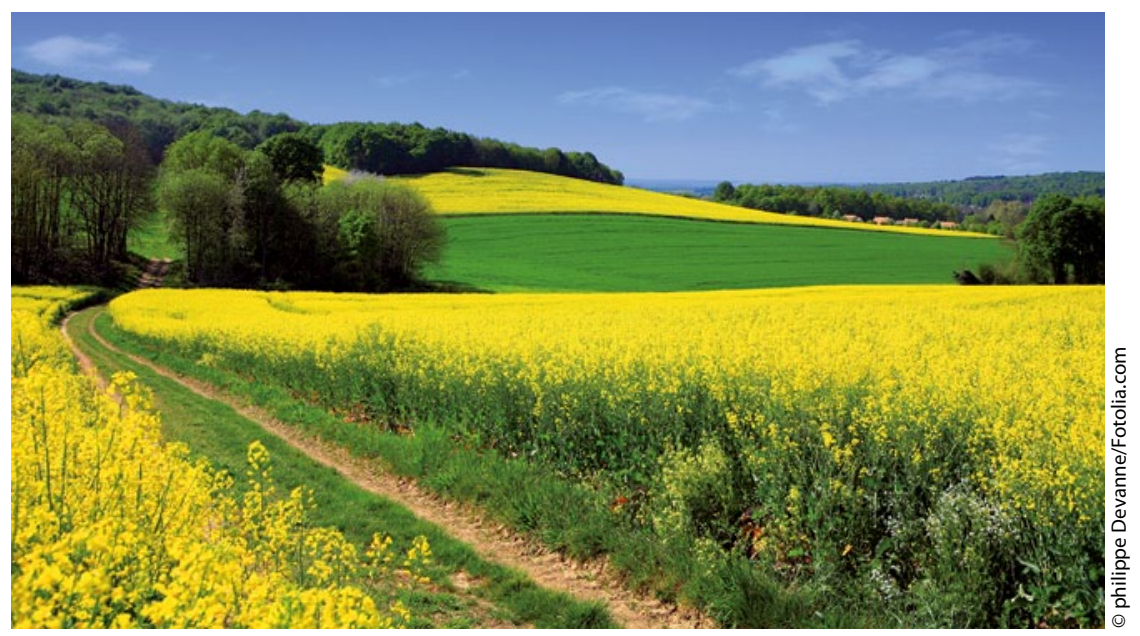

\section{Umdenken im Gesundheitsbau}

\section{„Salutogenetisches Design“ für Krankenhäuser}

- Nach Ansicht des Arztes und Architekten Alan Dilani beschäftige sich das Gesundheitswesen bis dato zu sehr mit pathogenetischen Faktoren, den Ursprüngen und der Behandlung von Krankheiten. Viel wichtiger sei es aber, sich auf gesundheitsfördernde Aspekte zu konzentrieren. Auf der internationalen Fachmesse Hospital Build Europe warb der Leiter der International Academy for Design and Health nach deren Angaben jüngst bei Gesundheitsmanagern für ein Umdenken im Gesundheitsbau. "Gesundheit ist nicht die Abwesenheit von Krankheit, sondern Wohlbefinden - nach dieser These sollten Architekten Häuser errichten", appellierte er an die Kongressbesucher. Nach Dilanis Vorstellung gehören zu einem gesundheitsfördernden Klinikgebäude ein lichtdurchfluteter Eingangsbereich, grüne Flächen, Wasserelemente, Orientierungshilfen durch Farben, in den Boden eingelassene Wegweiser, aber auch Kunst.,Ist die Zimmerdecke in einem Aufwachraum hübsch bemalt, können sogar Schmerzen der hier liegenden Patienten gelindert werden", erläuterte Dilani einen Vorzug seines „salutogenetischen Designs".
Matthias Wallenfels 